\title{
Laser-based techniques for a multidisciplinary action aimed at the restitutive restoration of $S$. Costanzo church in Ronciglione (Italy)
}

\author{
Valeria Spizzichino $^{1 \star}$, Luisa Caneve ${ }^{1}$, Massimiliano Ciaffi ${ }^{1}$, Roberta Fantoni ${ }^{1}$, \\ Massimo Francucci ${ }^{1}$, Massimiliano Guarneri ${ }^{1}$, Antonio Palucci ${ }^{1}$, Gaetano \\ Terranova $^{1}$, Franca Persia ${ }^{2}$, Angelo Tati ${ }^{2}$, Maria Fernanda Falcon Martinez ${ }^{3}$, \\ Chiara Giuffrida ${ }^{3}$, Francesca Scirpa ${ }^{3}$, Laura Bartoli ${ }^{4}$, Alessandro Zanini ${ }^{4}$
}

1 ENEA - Italian National Agency for New Technologies, Energy and Sustainable Economic Development, Via Enrico Fermi, 45, 00044 Frascati (Rome), Italy

2 ENEA - Italian National Agency for New Technologies, Energy and Sustainable Economic Development, Via Anguillarese, 301, 00123 Rome, Italy

3 Freelance conservator

4 El.En. S.p.A. Via Baldanzese, 1750041 Calenzano Firenze - Italy

* Corresponding author: valeria.spizzichino@enea.it

\begin{abstract}
In the framework of the COBRA (Conservation of Cultural Heritage through Radiation and Enabling Technologies) project a multidisciplinary study was carried out in order to restore the mural painting of the dome of the S. Costanzo church in Ronciglione (Italy). Large difficulties were met during the restitutive restoration of the original renaissance fresco decorating the dome, patronized by the Italian Ministry of Cultural Heritage (SABAP RM MET). In fact, two and sometimes three layers of paint, which lays on an extremely crumbled substrate were detected on the surface, in addition to large, deep and complex degradation forms with the presence of efflorescence. To solve such a critical situation, researchers, conservators and laser cleaning system suppliers collaborated to plan the most appropriate intervention. Laser-based diagnostics were employed to characterize different areas of the fresco surface before and after cleaning tests. In particular, ENEA offered its prototypes of LIF (Laser Induced Fluorescence) and RGB-ITR (Red Green Blue Imaging Topological Radar, an innovative 3D colour laser scanner) in addition to a laser Raman system and an infrared camera for thermographic measurements for both the material and degradation recognition and a micro-structural study. The results obtained allowed pointing at the most suitable restoration and conservation strategies. The cleaning tests were performed with both classical methods and lasers made available by El.En. group. Different operative parameters such as pulse duration, repetition rate, energy were tested. The laser employed made it possible to overpass the difficulties encountered by other cleaning techniques: degradation and painting layers were removed selectively, allowing bringing to light the original surface.
\end{abstract}

Keywords: Laser Induced Fluorescence, Raman spectroscopy, laser cleaning, Imaging Topological Radar 


\section{Introduction}

In order to face a diagnostic/conservation/ restoration project, a broad collaboration among different experts is needed. In particular, art historians, conservators, scientists and administrations (museum's managers, employees at departments of National Cultural Heritage) have to work in strict collaboration to obtain satisfactory results. In fact, their jobs and their expertise are complementary. Moreover, all of them have questions to ask and answers to offer. The term "archaeometry" was coined in 1958 as a name of the internal bulletin of the Research Laboratory for Archaeology and the History of Art of Oxford to provide an answer to the Humanist's questions: what is that? How was it realized? What is the material provenance? What kind of materials are used? In the same period, between the years 1957 and 1959, the first lasers were created and patented. Since then, archaeometry and lasers had developed on different ways up to 1970 s when their paths crossed [1]. Since that time, several spectroscopic and optical laser techniques have been applied to artworks and demonstrated their effectiveness, mainly as case studies and only for "scientific" purposes. The time is ripe for a more synergistic and multidisciplinary approach in artwork diagnostics and conservation. The availability of low cost and well-tuned laser instrumentation may help to fulfil this request [2-5]. In this context, ENEA (Italian National Agency for New Technologies, Energy and Sustainable Economic Development) is carrying out a project called COBRA (Conservation of Cultural Heritage through Radiation and Enabling Technologies), funded by Latium Region (lr13, n.1031), which is addressed to the transfer of innovative technologies and methodologies from research to $\mathrm{CH}$ end users and SME involved in the conservation. In the frame of the project COBRA, ENEA offers technologies and expertise in creating digital services, mobile and fixed laboratories to respond to end users' requirements and to the requests of the specialized market. The first case-study was a multidisciplinary action carried out in order to restore the mural painting of the dome of the S. Costanzo church in Ronciglione (Italy). To solve the large difficulties met during the restitutive restoration of the original renaissance fresco decorating the dome, researchers, conservators and suppliers of lasers for cleaning collaborated to plan the most appropriate intervention. Several areas of the treated surface were analysed and characterized by laser-based diagnostics before and after cleaning tests. LIF (Laser Induced Fluorescence) and Raman spectroscopy were applied to the recognition of both constituent materials and degradation forms. A micro-structural study was performed with an RGB-ITR (Red Green Blue Imaging Topological Radar). Thermal Infrared Technology was applied to detect humidity in the walls, detachments, fissures. Both classical chemical methods and lasers made available by El.En. group were tested for the cleaning of the degraded painted surface. After several evaluations, the most suitable laser and operation parameters were selected for every area. In the places where no other techniques worked, the laser cleaning allowed bringing to light the original surface.

The characteristics and critical issues of the dome decoration, details of the apparatus used and the first results obtained are presented in the following sections. 


\section{The decoration of the dome of S. Costanzo}

Historical documentation traces the church's origin back to the $16^{\text {th }}$ century. During its lifetime, the decorative setting has gone through many changes, and the current general appearance spans from the baroque style of the altar to the neoclassic one of the nave. The dome shared its fate: the ongoing restoration work is showing the visible coffered decoration of the $18^{\text {th }}$ century, concealing other more ancient decorations. A long history of infiltration and carelessness had produced several damages such as the decay of mortar, accretions, brittleness, losses and flaking. The paintings appeared to be in very poor condition, but yet several different and fine decorative pieces were still visible. An earlier overview highlighted the presence of two/three layers of paint, irregularly overlapped one to each other, laying on a plaster substrate extremely disaggregated and with the presence of efflorescence. In Fig. 1 examples of the deteriorated surfaces are presented.

On a disaggregated mortar, filled with soluble salt deposits and altered materials due to past infiltrations, there was a fresco with a glory of angels, of very fine manufacture, yet highly damaged and overpainted. It presented painting holes, secco paintings and strong decohesion. On such layer, there was a polychromy at lacunars motif, painted without any preparatory layer and engraving the decorative pattern directly on the fresco layer. In some areas, there was a third layer having another polychromy with the same lacunar motif of underlying layer. The degradation of the polychrome multi-layer was so dramatic that it could not sustain neither the pressure of a lancet nor the weight of a compress. Then, issues encountered in consolidation, in identification of biodeteriogens and of different pictorial techniques that were used required accurate investigations and the use of the least invasive techniques. Moreover, due to the large quantity of areas to be evaluated rapidly (see Fig. 2), fast imaging techniques were preferred in order to reduce the time of the analysis.

After the first phase of the study of the dome, of the decoration and of its degradation, the open questions were emphasised. In fact, to face the consolidation and the cleaning of the surface correctly, leading to the restitution of the original renaissance fresco, the conservators needed to know the techniques (secco or tempera) used for the outer layers, have knowledge regarding the presence of biodeteriogens, detachment and crack location. Moreover, since the first tests of consolidation by acrylic resins showed the lack of homogeneity in the distribution

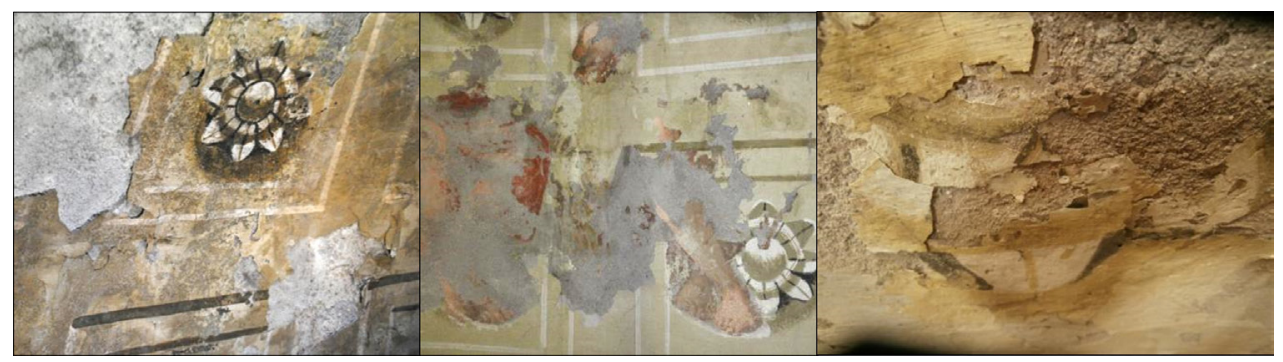

Fig. 1. Deterioration of decorate surface of the dome. 


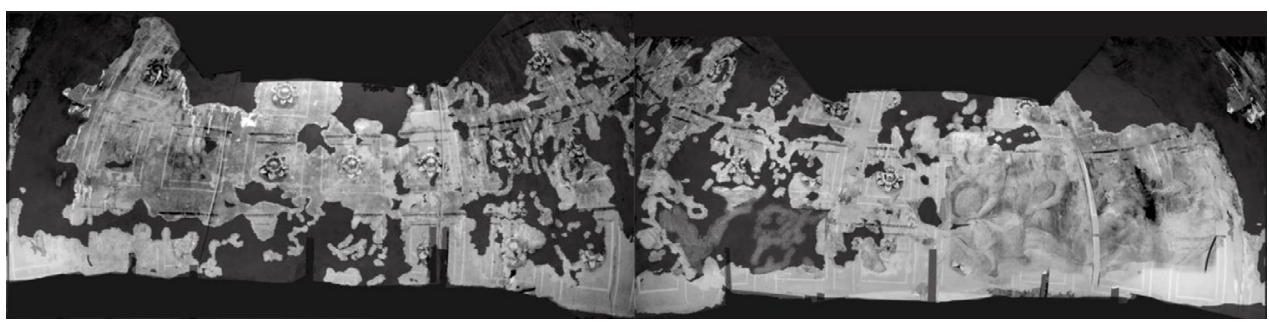

Fig. 2. The $360^{\circ}$ view of the dome showing the major intervention areas (in light gray). The circumference at $80 \mathrm{~cm}$ is $14 \mathrm{~m}$. The total dome area is $37 \mathrm{~m}^{2}$ of which $22 \mathrm{~m}^{2}$ with painting remains.

of the consolidants in the surface, their penetration level and migration directions had to be evaluated. Finally, the possibility to perform a laser cleaning and its efficiency were evaluated.

As in many real cases, referring to large artworks, the main requirements were the ability to work in situ (in uncontrolled experimental conditions), in quasi-real time conditions, in order to quickly find a solution to specific problems, mapping large areas, without sampling. To meet them, the techniques presented in next session were selected.

\section{Techniques}

For the characterization of constituent materials and degradation forms Laser Induced Fluorescence (LIF) and Raman spectroscopy were used $[5,6]$. For the latter, a portable $i$-Raman spectrometer (B\&W TEK Inc., USA) provided with a $785 \mathrm{~nm}$ excitation laser source and a CCD detector thermoelectrically cooled at $10^{\circ} \mathrm{C}$ was chosen. The spectrometer having a resolution of $3 \mathrm{~cm}^{-1}$ covers the spectral range $789-1048 \mathrm{~nm}$, corresponding to Raman shifts of $75-3200 \mathrm{~cm}^{-1}$. The excitation source is a GaAlAs solid state diode laser with a power scalable in the range 3-300 $\mathrm{mW}$. The laser power used during the measurements was set to a few milliwatts to prevent pigment photodecomposition. Typical acquisition conditions were 1 to 10 accumulations with exposure times of 1-5 s each. For measurements a probe, equipped with a quartz window and a spot diameter of $85 \mu \mathrm{m}$, was used. It has two optical fibres: the first is to deliver the excitation source and has a $105 \mu \mathrm{m}$ core, the second is for the collection and is characterized by a $200 \mu \mathrm{m}$ core. For LIF measurements a scanning prototype able to collect hyperspectral fluorescence images on large areas, realized at the ENEA in the Diagnostic and Metrology Laboratory of Frascati, was used. The system performs a point by point scanning, suitable for rapid investigations on large areas. A diode pumped Nd:YAG laser source is utilized to generate the UV radiation at $266 \mathrm{~nm}$ with repetition rate of $20 \mathrm{~Hz}$, pulse duration of $8 \mathrm{~ns}$ and energy up to $1.5 \mathrm{~mJ}$, which is well below the damage threshold previously tested on fresco's specimens. Each scan is monitored by a portable computer in which a specific program developed in LabView allows setting experimental parameters and controlling data acquisition. In the main control panel, data are shown both as $2 \mathrm{D}$ monochromatic images and LIF spectra, from $270 \mathrm{~nm}$ to 750 $\mathrm{nm}$ with a $2.5 \mathrm{~nm}$ spectral resolution, for each pixel. Additionally, the LIF scanning system can be utilized as hyperspectral analyser, with 
the laser switched off, to collect reflectance images upon the availability of an intense standard light source. For locating discontinuities and detachments thermovision and RGB-ITR (Red Green Blue Imaging Topological Radar) measurements were performed and compared. For thermographic measurements a commercial infrared camera FLIR T440 with frame rate of $60 \mathrm{~Hz}$, field of view $25^{\circ} \times 19^{\circ}$, a minimum focus distance of $0.4 \mathrm{~m}$, thermal sensitivity $<0.045^{\circ} \mathrm{C}$ at $30^{\circ} \mathrm{C}$, a focal plane array (FPA) uncooled microbolometer $320 \times 240$ pixels as a detector and a spectral range between 7.5 and $13 \mu \mathrm{m}$ was used. The RGB-ITR is a scanner prototype developed in DIM laboratory at ENEA Frascati: the main feature of this system is the capability to collect simultaneously both colour and structural information by the use of three amplitude modulated lasers and respective photodiodes [7].

\section{Diagnostic Results}

Measurements were performed mainly in the western and southern sectors of the dome, which appear to be the most interesting and rich in original fresco remains. With the LIF system some different areas of about $0.25 \mathrm{~m}^{2}$ each were scanned with a spatial resolution of about $1 \mathrm{~cm}$. This value is a compromise between the need of shortening acquisition times and those of providing answers to the specific questions asked by conservators. All collected spectra were processed and used to build fluorescence images, as the ones shown in Fig. 3. To generate every image, spectral data were filtered at the selected wavelength and normalized by the maximum obtained at such wavelength on the whole scan. In particular, in Fig. 3, fluorescence images at $300 \mathrm{~nm}$ and $700 \mathrm{~nm}$ are reported. They are of great importance because they are indicative of the presence of acrylic consolidants on the one hand and of photosynthetic microorganisms on the other, according to literature [8] and a database prepared thanks to laboratory research conducted on known samples. In this way, characteristics not visible by naked eye were highlighted. The obtained results confirmed the presence of preferential migration directions for consolidants injected in the surface for the consolidation action (Fig.3, centre), providing detailed maps which can help conservators in the evaluation of the consolidation technique.

The fluorescence spectra of the whole surface are dominated by the presence of large bands centred at $450 \mathrm{~nm}$ and $500 \mathrm{~nm}$ due to the presence of $\mathrm{CaCO}_{3}$ and $\mathrm{CaSO}_{4}$. No signals that can be referred to organic

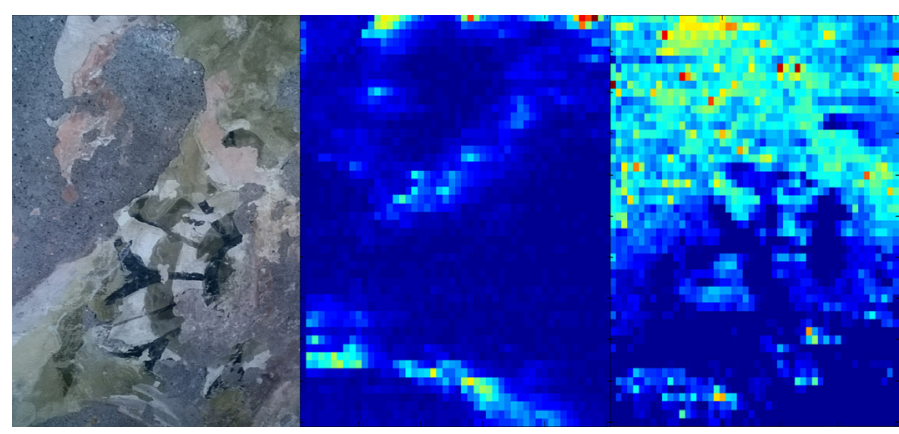

Fig. 3. An area examined by LIF: standard image in visible range (left), fluorescence map at $300 \mathrm{~nm}$, indicative of acrylic consolidants (centre), fluorescence map at $700 \mathrm{~nm}$, indicative of photosynthetic microorganisms (right). In the maps the colour is from blue for low intensities to red for higher intensities at the selected wavelength. 
binders, in agreement with a database built in laboratory from known samples prepared according to Cennino Cennini's recipes [9], were found on the surface. This observation would exclude the use of a tempera technique for the repainting of the dome, but rather it would suggest the use of lime painting. These results were confirmed by Raman spectroscopy, which allowed to recognize pigments and constituent materials. In the outer layers in the dark spots a black carbonaceous pigment from animal sources (ivory black or/and bone black) was individuated (Fig. 4 left) [10]. In the original fresco in red and pink areas, red ochre, mixed with $\mathrm{CaCO}_{3}$ and $\mathrm{CaSO}_{4} \times 2 \mathrm{H}_{2} \mathrm{O}$ was found (Fig. 4 right). Moreover, characteristic signals from calcium carbonate and sulphate were found on the whole surface without any signals that could be assigned to organic binders, in the limits of detections of the specific instrument used. In fact, spectral bands that can be assigned to aliphatic modes (near $3000 \mathrm{~cm}^{-1}$ and between 900 and $1500 \mathrm{~cm}^{-1}$ ) or to carboxylic group and unsaturations (1600-1800 $\mathrm{cm}^{-1}$ region) typical of most common historical binding media (fresh and aged) were not detected $[11,12]$.
The RGB-ITR was used for digitalizing before and after the laser cleaning intervention. This kind of digitalization had a dual purpose: the first was to have a historical memory of the drawings before the cleaning of the surface, the second to have two 3D multispectral maps of the interested areas for a semi-automatic detection of possible colour or structural modifications caused by the laser-cleaning intervention. This result was obtained by superimposing the data collected by different instruments (like LIF, RAMAN and thermography) on RGB-ITR $3 \mathrm{D}$ colorimetric meshes. The RGB-ITR was tested only on a limited portion of the dome: the rest of the structure was digitalized by the use of a low-cost structured light sensor called COBRAKIN. This latter sensor is also able to collect high definition photos by the use of a standard camera: this information was affected by the environmental lights, because it was impossible to create a photographic light setup. For calibrating these images the RGB-ITR colorimetric information will be used. An example of the images obtained is presented in Fig. 5. In this figure, a thermogram of a selected area (in the black box) is presented, too. Thermog-

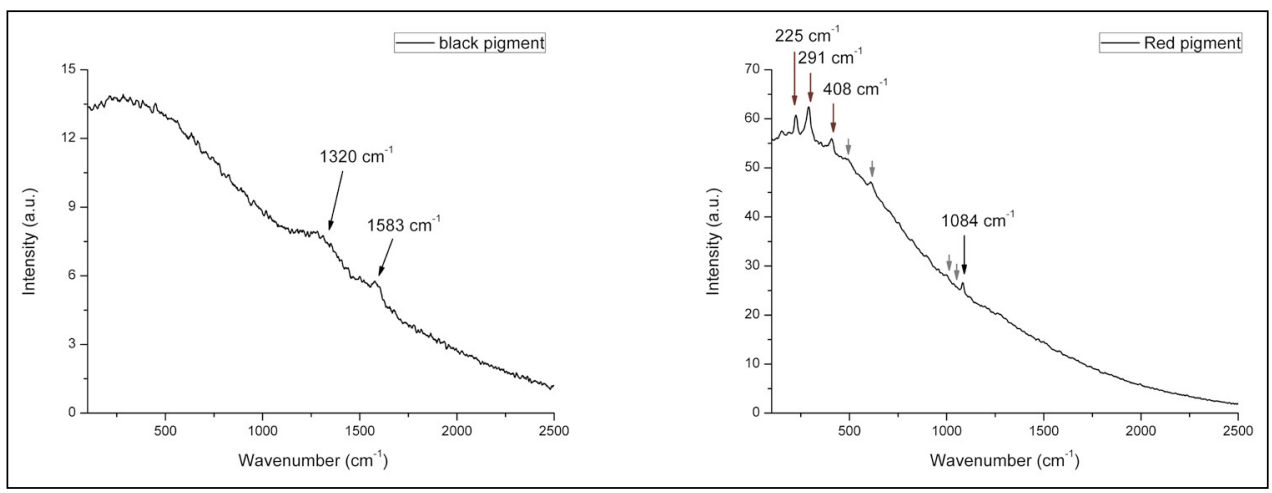

Fig. 4. Raman spectra for: black pigment in the outer layers (left) and for red pigment in the original fresco (right). In the latter Raman lines from calcite (at $1084 \mathrm{~cm}^{-1}$ ), red ochre (at 225, 291, $408 \mathrm{~cm}^{-1}$ ) [13] and $\mathrm{CaSO}_{4} \times \mathrm{H}_{2} \mathrm{O}(492,621,1007$, and $1135 \mathrm{~cm}-1)$ are clearly visible. 


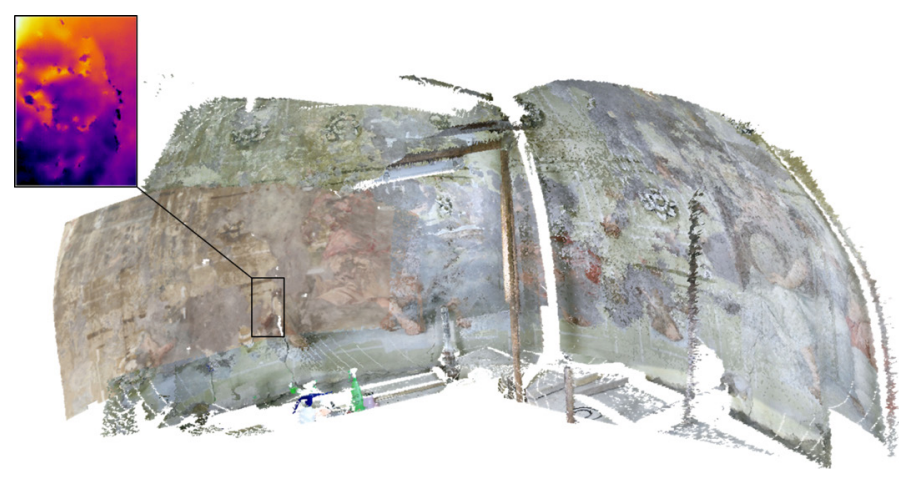

Fig. 5. on the left RGB-ITR scan (on the west sector of the dome) on the first tests with a new prototype, COBRAKIN. In the box a thermogram with a highlighted detachment area in white-yellow.

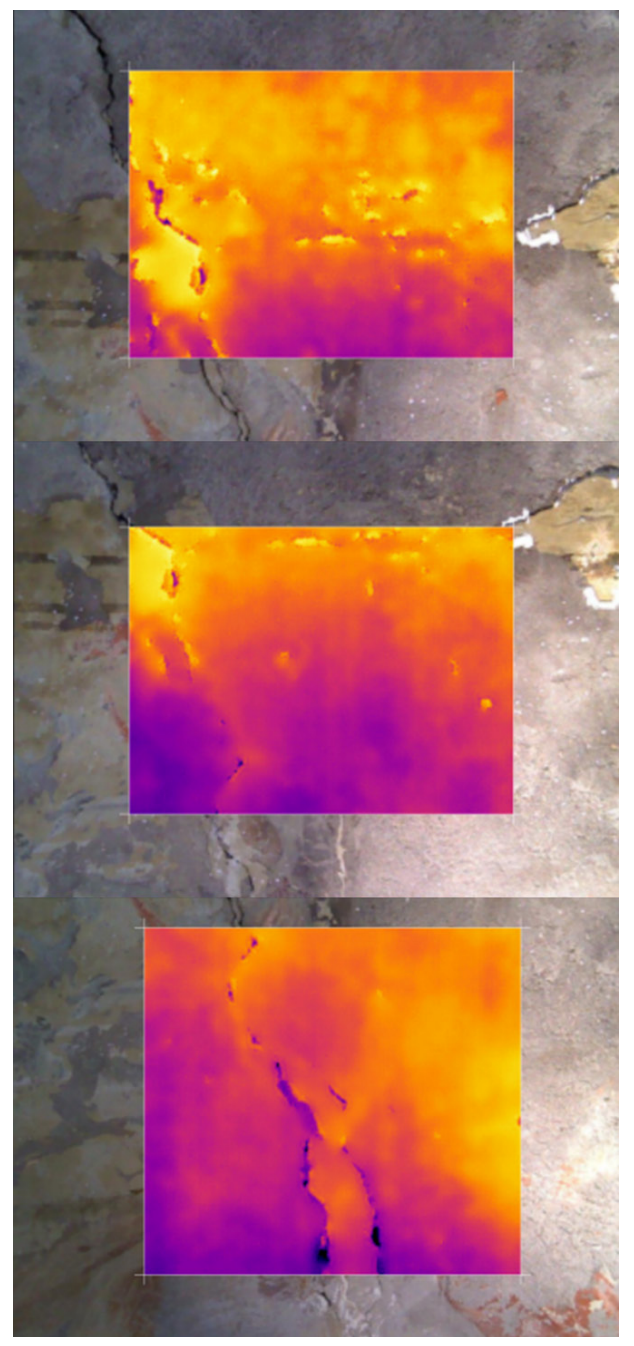

raphy revealed widespread detachments of the painted areas of the dome. They can be attributable to phenomena of crystallization of the soluble salts due to the presence of rainwater and/or the biological attack. The presence of overlapping layers of paint could triggered widespread detachments of the surface layers. External materials coming out from previous restorations can be the reason for the final degradation state. The images of the upper dome levels showed very critical conservation conditions due to rain water infiltration (Fig. 6).

\section{Laser cleaning}

The laser cleaning technique [14-16] was tested thanks to the collaboration with El.En. S.p.A. In particular, the system COMBO EOS 1000 was used. It is characterized by an emission at $1064 \mathrm{~nm}$ and two temporal regimes in one system: Short Free Running (SFR) mode, with a pulse duration ranging from 30 to 110 microseconds, and Long Q-switch (LQS) regime with 100ns. The energy ranges are 200-1400 $\mathrm{mJ}$ for the SFR mode and from $150 \mathrm{~mJ}$ to $450 \mathrm{~mJ}$ for the LQS mode.

$\leftarrow$ Fig. 6. Results of thermovision on the west sector, same area analysed by LIF and presented in Fig. 3 . 


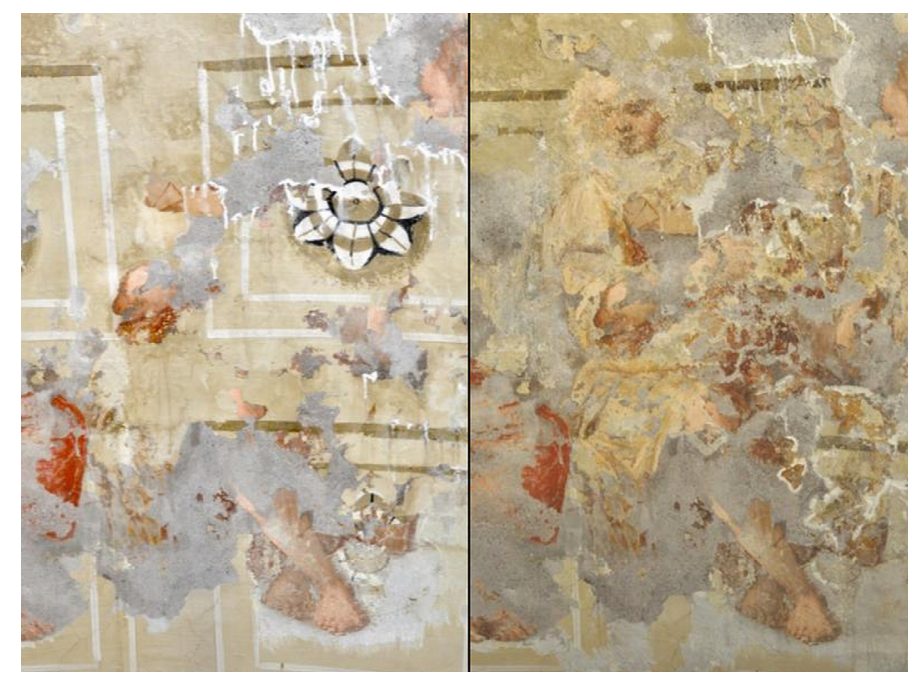

Fig. 7. Before (left) and after (right) the application of laser cleaning.
The selection of the operation parameters was particularly hard due to the high variability of impact points characteristics. In fact, the laser beam can impinge on areas with salts between fresco and the upper layers, on cohesive and tenacious layers, on fresco layer pulverized mixed with mortar, fresco layer damaged and patchy, on mortar, or on wide detachments. Moreover, chromatic selectivity was impossible because of the presence of polychromy in every different layer, which caused main difficulties. Nevertheless, after several tests the most suitable working conditions for every kind of problems were individuated. Both regimes were been used depending on the micro-area under study and for both of them the energy level was manually modified empirically to obtain the best result.

The successful outcomes of the laser cleaning are mainly due to a secondary spallation effect that allowed to remove micro-portions of the outer layers with high accuracy. An example of the obtained results is shown in Fig. 7. Here the same area of the dome before (left) and after (right) the laser cleaning is shown. As it is possible to notice on the left, originally a lacunar damaged motif is present and just some fragments of a human figure are visible (mainly feet at the bottom of the picture). The laser cleaning allowed discovering a whole cantor angel (clearly visible in the picture on the right). Thanks to this painstaking cleaning work several angel figures of good quality emerged.

Important results were obtained due to an accurate and slow work of restorers that cleared a large part of the surviving paintings from repainting with the use of the laser. As no other cleaning techniques could be successfully applied on the most part of the dome, the artwork was given up for lost up to the use of the laser cleaning. The frescoes in the dome of S. Costanzo proved to be a great surprise: there are just rare and sporadic documentary sources referring to it and no publications that remember the existence of this cycle. Further and deeper historic and artistic studies are being carried out by the SABAP RM MET.

After the removal of degradation and outer decoration layers, a laser cleaning 
tests were carried out on the pitting due to biological attack found on the original fresco. Moreover, in the areas previously cleaned by classical chemical manual methods, the EOS Combo removed also a residual thin layer of white patina. The remote colour measurements obtained by RGB-ITR on areas already treated with chemical approaches showed that the colours obtained after the laser cleaning are much more saturated than before the intervention, highlighting the ability of the laser cleaning to remove thin patinas, impossible to eliminate by means of other techniques.

The good performances of laser cleaning have now allowed other important interventions on the dome, first of all the consolidation of plaster and the refurbishment of the aesthetic appearance.

\section{Conclusions}

This work highlighted that the collaboration among different competences of people working on Cultural Heritage is necessary to achieve a correct and comprehensive restoration and conservation action. In fact, the collaboration between conservators, art historians and scientists allowed to verbalise open questions, to identify more suitable methodologies, to correctly read into the results, to reach complete answers. Different laser systems were used for diagnostics, documentation and cleaning of the dome of S. Costanzo church in Ronciglione (Italy). In particular, a LIF scanning system and Raman spectroscopy were applied to characterize the materials (both original and used during restoration actions) and to obtain information about techniques used for the different painting layers. Moreover, the ability of the LIF prototype to produce fluorescence images allowed to map bioattacks and preferential migration directions of consolidants used by restorers. The EOS Combo system by El.En. was used successfully for the effective cleaning of the surface, succeeding where classical mechanical and chemical cleaning methods had failed. The RGB-ITR prototype provided both the mapping of cracks and detachments in collaboration with thermography, and a 3D colour documentation for technical and media purposes before, during and after the cleaning of the dome.

\section{Acknowledgements}

The authors would like to thank dr Luisa Caporossi and dr Federica di Napoli Rampolla, from the Ministry of Cultural Heritage SABAP RM MET, dr Pietro Paolo Lateano, and Silvio Iacomi for making this work possible. This research was supported by the Latium Region under grant agreement lr13, n.1031.

\section{References}

[1] J.F. Asmus, C.G. Murphy, W.H. Munk, "Studies on the Interaction of Laser Radiation with Art Artifacts", in: Developments in Laser Technology II, San Diego, USA, August 27, 1973, Proceedings of SPIE 0041, 1974, DOI: $10.1117 / 12.953831$.

[2] A. Nevin, G. Spoto, D. Anglos, "Laser spectroscopies for elemental and molecular analysis in art and archaeology", Applied Physics A, 106 (2012) 339-361, DOI: 10.1007/s00339-011-6699-z.

[3] G. Ricci, L. Caneve, D. Pedron, Nadine Holesch, E. Zendri, "A multi-spectroscopic study for the characterization and definition of production techniques of German ceramic sherds", Microchemical Journal 126 (2016) 104-112, DOI: 10.1016/j.microc.2015.12.009.

[4] V. Spizzichino and R. Fantoni, "Laser induced breakdown spectroscopy in archeometry: a review of its application and future perspectives", Spectrochimica Acta B, 99 (2014), 201-209, DOI: 10.1016/j. sab.2014.07.003. 
[5] D. Bersani and P. P. Lottici, "Raman spectroscopy of minerals and mineral pigments in archaeometry" Journal of Raman Spectroscopy, 47 (2016) 499-530.

[6] J.L. Kinsey, "Laser Induced Fluorescence”, Annual Reviews Physical Chemistry 28 (1977) 349-372, DOI: 10.1146/annurev.pc.28.100177.002025.

[7] M. Guarneri, M. Ferri De Collibus, G. Fornetti, M. Francucci, M. Nuvoli, and R. Ricci, "Remote colorimetric and structural diagnosis by RGBITR color laser scanner prototype", Advances in Optical Technologies, 2012 (2012) 1-6, DOI: 10.1155/2012/512902.

[8] B. Park, R. Lu, Hyperspectral Imaging Technology in Food and Agriculture, Springer, New York 2015, DOI: 10.1007/978-1-4939-2836-1.

[9] V. Spizzichino, L. Caneve, R. Fantoni F. De Nicola, "Spectral database of Renaissance fresco pigments by LIBS, LIF and colorimetry", in: Proceedings of the $3^{\text {rd }}$ Balkan Symposium on Archaeometry, Bucharest, Romania, October 29-30, 2012, R. Rãdvan, S. Akyuz, M. Simileanu, V. Dragomir (Eds.) Integra natura et Omnia - INOE Bucharest 2013, pp. 9-16, http://certo. inoe.ro/watch/images/proceedings.pdf (accessed 16.06.2017).

[10] C. Lofrumento, M. Ricci, L. Bachechi, D. De Feo and E.M. Castellucci, "The first spectroscopic analysis of Ethiopian prehistoric rock painting", Journal of Raman Spectroscopy 43 (2012), 809-816, DOI: $10.1002 /$ jrs.3096.

[11] P. Vandenabeele, B. Wehling, L. Moens, H. Edwards, M. De Reu, G. Van Hooydonk, "Analysis with micro-Raman spectroscopy of natural organic binding media and varnishes used in art", Analytica Chimica Acta, 407 (2000) 261-274, DOI: 10.1016/ S0003-2670(99)00827-2.

[12] S.A.Centeno, "Identification of artistic materials in paintings and drawings by Raman spectroscopy: some challenges and future outlook", Journal of Raman Spectroscopy, 47 (2016) 9-15, DOI: 10.1002/ jrs.4767.

[13] D. Bikiaris, Sister Daniilia, S. Sotiropoulou, O. Katsimbiri, E. Pavlidou, A.P. Moutsatsou, Y. Chryssoulakis, "Ochre-differentiation through micro-Raman and micro-FTIR spectroscopies: application on wall paintings at Meteora and Mount Athos, Greece", Spectrochimica Acta Part A 56 (2000) 3-18, DOI: 10.1016/S1386-1425(99)00134-1.

[14] S. Siano, J. Agresti, I. Cacciari, D. Ciofini, M. Mascalchi, I. Osticioli, A.A. Mencaglia, "Laser cleaning in conservation of stone, metal, and painted artifacts: state of the art and new insights on the use of the Nd:YAG lasers", Applied Physics A 106 (2012) 419-446, DOI: 10.1007/s00339-011-6690-8.

[15] N.Arnold, “Theoretical description of dry laser cleaning" Applied Surface Science 208-209 (2003) 15-22, DOI: 10.1016/S0169-4332(02)01278-3.

[16] A. Sarzyński, K. Jach, and J. Marczak, “Comparison of Wet and Dry Laser Cleaning of Artworks", in: Proceedings of LACONA VI Lasers in the Conservation of Artworks, Vienna, Austria, Sept. 21-25, 2005 M.J. Nimmrichter, W. Kautek, M. Schreiner (Eds.), Proceedings in physics 116 (2007), DOI: 10.1007/978-3-540-72130-7_19. 